\title{
Towards a conceptual framework demonstrating the effectiveness of audiovisual patient descriptions (patient video cases): a review of the current literature
}

\author{
Damian Roland ${ }^{1 *}$, Tim $_{\text {Coats }}{ }^{1}$ and David Matheson ${ }^{2}$
}

\begin{abstract}
Background: Technological advances have enabled the widespread use of video cases via web-streaming and online download as an educational medium. The use of real subjects to demonstrate acute pathology should aid the education of health care professionals. However, the methodology by which this effect may be tested is not clear.

Methods: We undertook a literature review of major databases, found relevant articles relevant to using patient video cases as educational interventions, extracted the methodologies used and assessed these methods for internal and construct validity.

Results: A review of 2532 abstracts revealed 23 studies meeting the inclusion criteria and a final review of 18 of relevance. Medical students were the most commonly studied group (10 articles) with a spread of learner satisfaction, knowledge and behaviour tested. Only two of the studies fulfilled defined criteria on achieving internal and construct validity. The heterogeneity of articles meant it was not possible to perform any meta-analysis.

Conclusions: Previous studies have not well classified which facet of training or educational outcome the study is aiming to explore and had poor internal and construct validity. Future research should aim to validate a particular outcome measure, preferably by reproducing previous work rather than adopting new methods. In particular cognitive processing enhancement, demonstrated in a number of the medical student studies, should be tested at a postgraduate level.
\end{abstract}

Keywords: Patient video clips, Methodology, Evaluation, Educational intervention

\section{Background}

There are a plethora of educational programmes and implementation strategies aimed at improving the quality of care delivered by health care professionals. A number of these are delivered via information technology systems with the use of video as an educational medium well established [1-3]. A new educational tool, that has become possible through multimedia advances in the last decade, is the audio-visual demonstration of signs

\footnotetext{
* Correspondence: dr98@le.ac.uk

'Emergency Medicine Academic Group, Emergency Department secretaries c/o Elizabeth Cadman-Moore, Leicester Royal Infirmary, Leicester LE1 5WW, UK

Full list of author information is available at the end of the article
}

and symptoms in patients, referred to as Patient Video Cases or PVCs [4]. They are easily displayed via online platforms, are widely used, and have been endorsed by the National Patient Safety Agency [5] as an example of good practice. However there is little academic study of their effectiveness. Given the financial pressures affecting all health care agencies, it is important to know if these resource intensive e-learning strategies give demonstrable benefit to patients or health care professionals.

Theoretical constructs exist to evaluate interventions designed to improve clinical performance, but no single approach is followed, due to the wide range of individual and organisational factors that affect the outcomes before, during and after the intervention [6]. Kirkpatrick's 
training evaluation is defined by four distinct levels of outcome to be approached in a stepwise fashion [7]. The four key domains of the Kirkpatrick model are learner satisfaction, learner knowledge, learner behaviour change and organisational change. Although others have argued contextual factors not classified under these domains may be significant [6], the Kirkpatrick model still remains a valid methodology with systematic reviews using the process to examine training effectiveness [8]. A healthcare relevant modification of the Kirkpatrick model has been used in a study of inter-professional education in health and social care [9]. When using the Kirkpatrick model, or other relevant frameworks for assessing an educational or training intervention, the outcome measures and the methodology by which they are obtained must be valid. The concepts of internal and construct validity are classifications with direct relevance to outcome measures and are components of methodological quality used by the Campbell Collaboration [10,11].

i. Internal Validity is the extent to which the intervention can reliably be ascribed to have affected the change

ii. Construct Validity relates to the association between the concept being investigated and the measures used to test it i.e. does the data collected accurately reflect the outcome measure chosen?

Other forms of validity exist but are not directly relevant to the quality of the outcome measures chosen, for example good external validity would imply that using PVCs could be beneficial in different populations, but would not give any information if the initial outcome measure was fit for purpose.

The aim of this work is to answer the question "What is the validity and quality of outcome measures that have been used to evaluate interventions based on PVCs?". This literature review will be used to identify which outcome measures are most valid in the assessment of the clinical effectiveness of an intervention based on PVCs. It will also help identify areas where more methodological research is needed to enable future studies to demonstrate high internal and construct validity.

\section{Methods}

This review was performed over three stages, the first stage collating relevant literature followed by individual study quality appraisal in stage two with a summation of the overall validity of the studies.

\section{Stage one}

Stage one identified literature relevant to the use of PVCs in health care settings. The definition of Health Care Settings used was; 'any location or environment where students or graduates are practising or learning medicine.' The definition of a PVC was; 'any pre-recorded or live video footage of a patient used for the purposes of demonstrating a sign or symptom'. It did not include footage recorded for the purposes of educating other patients or families. Inclusion criteria were:

i. Humans

ii. The study described the use of PVCs in a training, educational (undergraduate or postgraduate), implementation capacity or environment.

As PVCs relate to demonstration of signs and symptoms in patients, studies using video to demonstrate verbal communication, non-lexical utterances or solely history taking between a patient and doctor or patient and patient were excluded as were non-English language papers which could not be translated. The full literature search was developed in conjunction with a senior NHS Librarian and is available on request. The following general search terms were used (Video* OR Video record* OR video clip OR digital* record* OR analogue recording OR patient video clip) and (Educat* OR Train* OR learn* OR teach* OR inservice training). The following databases were searched: Medline, British Nursing Index (BNI), EMBASE, Health Management Information Consortium (HMIC), CINAHL, NIHR Health Technology Assessment Programme (HTA), Database of Abstracts of Reviews of Effects (DARE), Scopus, The Cochrane Library and the Education Resources Information Centre (ERIC). Internet search engines and NHS evidence were used to identify publications or articles related to the search terms. The search strategy was not limited to any particular research methodology used in the articles. The last search performed was $27 \mathrm{th}^{\text {th }}$ July 2012 by the principal author. In all phases of the study any uncertainty as to classification or indexing of information was discussed with the collaborating authors.

Articles with a relevant abstract (any detail relating to the recording and utilisation of video clips of patients) had a complete paper review (as did any abstracts in which there was uncertainty about inclusion potential). Information on aim, health care user, educational purpose, modified Kirkpatrick training level domain, type of study, outcome measure and conclusions was extracted from each paper as shown in Table 1. The Educational purpose was subdivided into three categories:

\section{Stage two}

To enable objective review of articles to determine the aspects of validity under study the following domains were used which represent features reducing the internal validity of studies. They have been amended from the list described by Farrington [12]. This work was chosen as it 
Table 1 Studies by Healthcare professional grouping

is based on Cook and Cambell's original work on methodological quality. Although other methodologies of analysis are available this is a widely used and accepted process which allows for an objective process to be applied.

1. Selection: Does the outcome measure allow for control between groups?

2. History: Does the outcome measure allow for the effects caused by some event occurring at the same time as the intervention?

3. Maturation: Does the outcome measure allow for natural progression in learning and knowledge?

4. Instrumentation: Is the outcome measure reproducible?

5. Testing: Does the outcome measure itself affect the results?

6. Differential attrition: Can the outcome measure control for differing numbers of participants in control or experimental groups (if present) or large drop out rates.

The extraction of information was undertaken by the principal author.

\section{Stage three}

Once this process had occurred a number of more global questions were asked of each paper to determine whether the article's author had evaluated the outcome methods they had chosen and allow an assessment of the construct validity of the study.

a) How was the choice of outcome measure justified?

b) Did the choice determine the results the study aims to investigate?

c) To what extent were the writers aware of the disadvantages as well as the advantages of the outcome measures chosen?

d) How did they overcome the disadvantages?

\section{Results}

Figure 1 shows the flow of journals from the initial search to the final selection of articles. The types of healthcare professionals studied is demonstrated in Table 1 and the number of studies classified by educational purpose and Kirkpatrick level shown in Table 2. Two studies evaluated both undergraduate and basic postgraduate trainees leading to a total of 21 studies of health care professional groups and two studies evaluated both learner knowledge and learner behaviour leading to a total of 20 studies of the relevant Kirkpatrick level.

The purpose of this work was to be as inclusive as possible so as to capture all outcome measures used. Although twenty-two articles (twenty-three studies) underwent a thorough analysis in stage two, half of these require further clarification as to the reasons for their inclusion. These articles were all reviewed by all three authors and a collaborative decision reached on their inclusion. Under the inclusion criteria it had not been the intention to include animal studies in the protocol. However one, in the field of veterinary medicine [13], studied PVCs in precisely the context human patients clips would be used with an accompanying relevant and feasible methodology. It has been included in the final review as it was decided methodology rather than context was being investigated. The search was repeated removing the 'human only' limitation but no other veterinary journals of relevance were found.

One study examining an intervention to improve the physical examination component of a medical student exam via a web-based video did not specifically use abnormal or normal clinical signs [14]. The study looked at outcomes across a whole year group in a before and after cohort design. This study has been included as the methodology could have been easily used in a PVCrelated intervention. A study using video to demonstrate a specific clinical examination was also included although it could be argued that the precise aim of the 


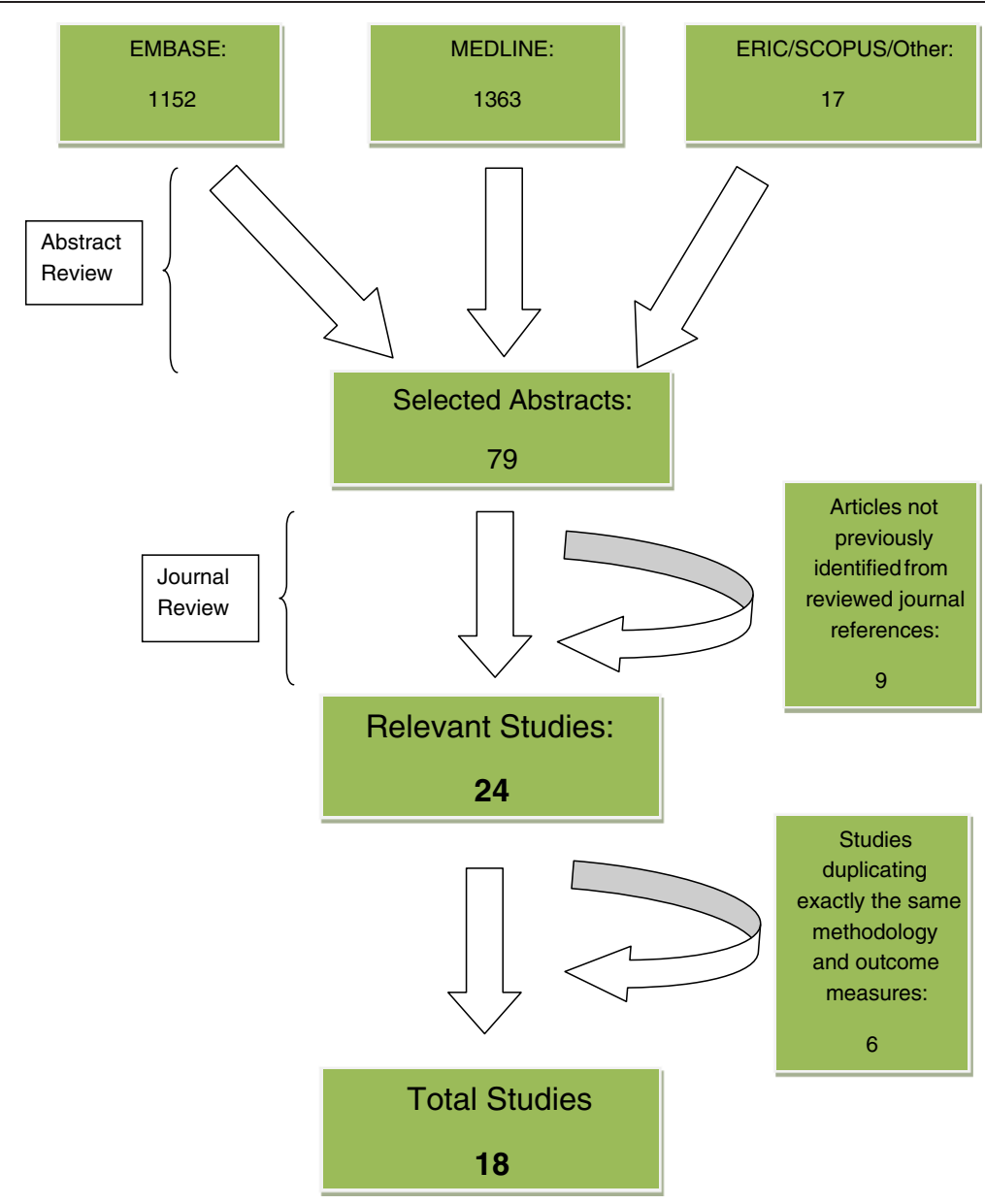

Figure 1 Literature Search Flow Diagram.

tool was not demonstrating specific clinical signs but a methodology of elucidating them. The methodology used, a Solomon four-group design [15], was considered relevant to defining robust outcome measures in future PVC studies.

Finally six studies [16-21], although in different patient groups (ankylosing spondylitis, rheumatoid arthritis, fibromyalgia) used exactly the same methodology as two initial studies into osteoarthritis by the same investigators. These were studies in the validation of an examination methodology in both medical students and consultants. Although the actual data was different, the papers used exactly the same introduction, methods and discussion. In terms of the narrative review, these eight journal articles represent only one methodological approach in two different cohorts of participants. It was felt due to the lack of difference in the wording of the arthritic publications these should be considered as two studies, one representing undergraduates and the other

Table 2 Classification of studies

\begin{tabular}{llll}
\hline Educational purpose & Number of studies & Kirkpatrick level & Number of studies \\
\hline Knowledge Gain & 8 & Learner Reaction (level 1) & 5 \\
Testing Methods & 3 & Learner Knowledge (2a) & 0 \\
Patient Examination Skills & 5 & Learner Knowledge (2b) & 8 \\
Overall Clinical Care & 2 & Learner Behaviour & 7 \\
Total & $\mathbf{1 8}$ & Total & $\mathbf{2 0}$ \\
\hline
\end{tabular}


Table 3 Identification of health care settings in which educational patient video clips have been utilised

\begin{tabular}{|c|c|c|c|c|c|c|c|}
\hline Paper & Aim or hypothesis & $\begin{array}{l}\text { Health care } \\
\text { user }\end{array}$ & $\begin{array}{l}\text { Educational } \\
\text { purpose }\end{array}$ & $\begin{array}{l}\text { Kirkpatrick } \\
\text { level }\end{array}$ & $\begin{array}{l}\text { Type of } \\
\text { study }\end{array}$ & $\begin{array}{l}\text { Outcome } \\
\text { methodology }\end{array}$ & Conclusions \\
\hline $\begin{array}{l}\text { Using interactive } \\
\text { video to add } \\
\text { physical assessment } \\
\text { data to computer } \\
\text { based patient } \\
\text { simulations [22] }\end{array}$ & $\begin{array}{l}\text { Interactive video in } \\
\text { patient simulations } \\
\text { improves the } \\
\text { learning experience } \\
\text { [Not formally Stated } \\
\text { in paper] }\end{array}$ & $\begin{array}{l}\text { Basic } \\
\text { Postgraduate } \\
\text { Training }\end{array}$ & $\begin{array}{l}\text { Overall } \\
\text { Clinical Care }\end{array}$ & $\begin{array}{l}\text { Learner } \\
\text { Behaviour } \\
\text { (level 3) }\end{array}$ & Comparative & $\begin{array}{l}\text { Chart Review of } \\
\text { interview and } \\
\text { physical } \\
\text { examination } \\
\text { description of } \\
\text { actual patients } \\
\text { versus simulation } \\
\text { performance }\end{array}$ & $\begin{array}{l}\text { Good to fair } \\
\text { agreement on } \\
\text { overall comparison } \\
\text { (kappa=0.72) and } \\
\text { physical } \\
\text { examinations } \\
\text { (kappa }=0.7 \text { ) }\end{array}$ \\
\hline $\begin{array}{l}\text { The development of } \\
\text { shared cognition in } \\
\text { paediatric residents } \\
\text { analysing a patient } \\
\text { video versus a } \\
\text { paper patient case } \\
\text { [23] }\end{array}$ & $\begin{array}{l}\text { Will supplementing } \\
\text { a written case } \\
\text { vignette by a PVC } \\
\text { as opposed to an } \\
\text { equivalent paper } \\
\text { case increase shared } \\
\text { cognition as } \\
\text { measured by the } \\
\text { frequency of }\end{array}$ & $\begin{array}{l}\text { Medical } \\
\text { Students }\end{array}$ & $\begin{array}{l}\text { Knowledge } \\
\text { Gain }\end{array}$ & $\begin{array}{l}\text { Leaner } \\
\text { Knowledge } \\
\text { (level 2b) }\end{array}$ & $\begin{array}{l}\text { Cluster } \\
\text { Randomised } \\
\text { Control Trial }\end{array}$ & $\begin{array}{l}\text { Identification and } \\
\text { frequency of } \\
\text { collaborative and } \\
\text { individual concept } \\
\text { links }\end{array}$ & $\begin{array}{l}\text { The video group } \\
\text { showed a } \\
\text { significant increase } \\
(p<0.01) \text { in } \\
\text { collaborative } \\
\text { concept links but } \\
\text { not in individual } \\
\text { concept links after } \\
\text { watching the video }\end{array}$ \\
\hline
\end{tabular}

$\begin{array}{ll}\text { Enhancing } & \text { (1) How does the } \\ \text { diagnostic accuracy } & \text { level of diagnostic } \\ \text { among non-experts } & \text { accuracy evolve } \\ \text { through use of } & \text { through an } \\ \text { video cases [24] } & \text { interactive } \\ & \text { teamwork approach } \\ & \text { using PVCs? } \\ & \text { (2) Does the level of } \\ & \text { diagnostic accuracy } \\ & \text { differ between non- } \\ & \text { experts and } \\ & \text { experts? }\end{array}$

Specialist Knowledge Postgraduate Gain Knowledge Measures (level 2b) Design

formation in the context of collaborative learning about movement disorders....?

experts?

Introduction of
patient video clips
into computer-
based testing:
Effects on item
statistics and
reliability estimates
[25]
[25]

Video-based test questions: A novel means of evaluation [26]

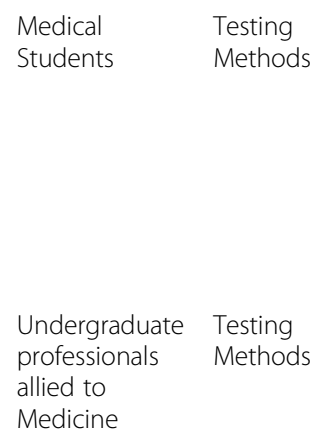

(1)

Questionnaire utilising repeated measures ANOVAS

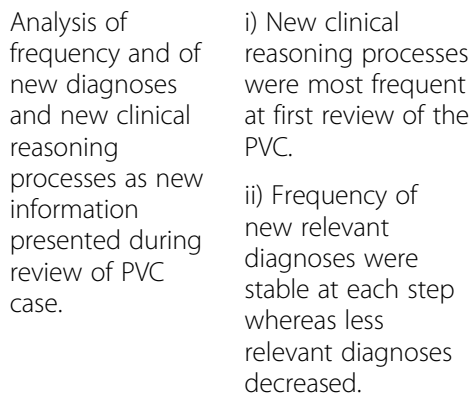

i) New clinical reasoning processes were most frequent at first review of the PVC.

ii) Frequency of new relevant diagnoses were stable at each step whereas less relevant diagnoses decreased.

Median Item statistics and reliability estimates for test items

iii) Relevant clinical reasoning was significantly higher amongst nonexperts compared with experts at the small group discussion and think aloud procedure with content expert.

Overall, video-based questions had comparable difficulty and discrimination compared to analogous textbased questions.

Students preference between videobased and multiple choice questions 
Table 3 Identification of health care settings in which educational patient video clips have been utilised (Continued)

Video-based test
questions: A novel
means of evaluation [26]

\begin{abstract}
A comparison of critical thinking in groups of third-year medical students in text, video, and virtual PBL case modalities [27]

Critical Thinking, as exemplified by the discourse among students during group discussion, differs among groups receiving the same case with the same facilitator in one of three formats.

Comparison of text
and video cases in a postgraduate problem-based learning format [28]

the frequency of
data exploration,
theory building and
evaluation and
metareasoning than
would be a
achieved by a paper
case.
\end{abstract}

Use of animationenhanced video clips for teaching To gather feedback regarding the Animated Breathing abnormal breathing Pattern Videotape patterns [13]

Using web-based video to enhance physical examination skills in medical students [14]

To measure changes in first year students'

performance of

physical

examinations on

standardized [sic]

patients after

implementation of a

web-based

curriculum

Teaching the plantar reflex [15]

To test to efficacy of Medical

video-tape in the Students

evaluation of the

planter response

$\begin{array}{lll}\text { Undergraduate } & \text { Testing } & \text { Learner } \\ \text { professionals } & \text { Methods } & \text { Knowledge } \\ \text { allied to } & & \text { (level 2b) } \\ \text { Medicine } & & \end{array}$

Medical

Students

Knowledge

Gain

Medical

Students

$3^{\text {rd }}$ year

Veterinary

Students,

House Officers

and Faculty,

Qualified

Veterinarians

Medical

Students

Patient

Examination

Leaner

reaction

Skills

(level 1)
(2)

Observational

comparative

study (One

group

informed

about video

questions the

other not)

Three way

comparative

study (face-2-

face with text

face-2-face

with video,

virtual with

video)

\section{Learner}

Knowledge

(level 2b)

Randomised

comparative

study of video

versus text

cases

Exam scores in 12 Students informed

video-based items about video clips

correctly answered more video based

items

Critical thinking

discourse analysis

The virtual groups

had the highest

critical-thinking

ratio. Except for the problem-

identification stage,

the video groups

had higher ratios

that the text groups

did.

Frequency of pre- The verbal

defined clause interaction showed

categories

statistically

significant

improvements in

data exploration

theory building and

theory evaluation

after the video case

Questionnaire Usefulness and

Satisfaction

Uniformly positive

responses

Patient

Examination

Skills

Learner

Behaviour

(level 3)

Before and

After Cohort

Outcome

study

Patient Learner

Examination

Skills
Behaviour

(level 3)
Solomon Four

Group Design

- TwO

experimental

and control

groups (with

and without

entrance test) (i) Percent correct score in physical exam item checklist

(ii)Mean score on physical exam process instrument
Students on Webbased curriculum had higher level of competency and reduction in poor performance levels
Correct judgement Small nonof graded

presence of clinical difference between sign

experimental and control groups [evidence of sampling error]. If analysis was restricted to students who performed an entrance test there was a statistical significance in favour of the video group. 
Table 3 Identification of health care settings in which educational patient video clips have been utilised (Continued)

\begin{tabular}{|c|c|c|c|c|c|c|c|}
\hline \multirow{4}{*}{$\begin{array}{l}\text { A videotape-based } \\
\text { training method for } \\
\text { improving the } \\
\text { detection of } \\
\text { depression in } \\
\text { residents of long- } \\
\text { term care facilities } \\
\text { [29] }\end{array}$} & \multirow{4}{*}{$\begin{array}{l}\text { Does a training } \\
\text { programme } \\
\text { involving video } \\
\text { based scenarios } \\
\text { improve nursing } \\
\text { staffs' detection of } \\
\text { depression within } \\
\text { long-term care } \\
\text { facilities? [Not } \\
\text { formally stated by } \\
\text { the paper] }\end{array}$} & \multirow{4}{*}{$\begin{array}{l}\text { Professionals } \\
\text { allied to } \\
\text { Medicine }\end{array}$} & \multirow[t]{4}{*}{$\begin{array}{l}\text { Overall } \\
\text { Clinical Care }\end{array}$} & \multirow{4}{*}{$\begin{array}{l}\text { Learner } \\
\text { Knowledge } \\
\text { (level 2b) } \\
\text { and Learner } \\
\text { Satisfaction } \\
\text { (level 3) }\end{array}$} & \multirow{4}{*}{$\begin{array}{l}\text { Parallel group } \\
\text { delayed } \\
\text { intervention } \\
\text { design. }\end{array}$} & $\begin{array}{l}\text { (I) Videotape } \\
\text { vignette test }\end{array}$ & \multirow{3}{*}{$\begin{array}{l}\text { Significant increase } \\
\text { in performance in } \\
\text { the intervention } \\
\text { group which was } \\
\text { maintained for at } \\
\text { follow up for both } \\
\text { vignette and } \\
\text { written test. }\end{array}$} \\
\hline & & & & & & (ii) Written Test & \\
\hline & & & & & & \multirow[t]{2}{*}{$\begin{array}{l}\text { (iii) Course } \\
\text { evaluation } \\
\text { questionnaire }\end{array}$} & \\
\hline & & & & & & & $\begin{array}{l}\text { Good levels of } \\
\text { satisfaction on } \\
\text { questionnaire }\end{array}$ \\
\hline $\begin{array}{l}\text { Advantages of } \\
\text { video trigger in } \\
\text { problem-base } \\
\text { learning [30] }\end{array}$ & $\begin{array}{l}\text { The reasons behind } \\
\text { preferences for } \\
\text { video triggers or } \\
\text { paper cases in } \\
\text { students and } \\
\text { facilitators who are } \\
\text { accustomed to } \\
\text { paper cases. }\end{array}$ & $\begin{array}{l}\text { Medical } \\
\text { Students }\end{array}$ & $\begin{array}{l}\text { Knowledge } \\
\text { Gain }\end{array}$ & $\begin{array}{l}\text { Learner } \\
\text { Reaction } \\
\text { (level 1) }\end{array}$ & Questionnaire & $\begin{array}{l}\text { Usefulness and } \\
\text { Satisfaction }\end{array}$ & $\begin{array}{l}\text { Video triggers were } \\
\text { preferred by both } \\
\text { students and } \\
\text { facilitators over } \\
\text { paper cases in } \\
\text { Problem Based } \\
\text { Learning }\end{array}$ \\
\hline $\begin{array}{l}\text { A triangulated } \\
\text { approach to the } \\
\text { assessment of } \\
\text { teaching in } \\
\text { childhood epilepsy } \\
\text { [31] }\end{array}$ & $\begin{array}{l}\text { Evaluation of } \\
\text { participant } \\
\text { perceptions of } \\
\text { learning }\end{array}$ & $\begin{array}{l}\text { Medical } \\
\text { Student s and } \\
\text { Basic } \\
\text { Postgraduate } \\
\text { Training }\end{array}$ & $\begin{array}{l}\text { Knowledge } \\
\text { Gain }\end{array}$ & $\begin{array}{l}\text { Learner } \\
\text { Reaction } \\
\text { (level 1) }\end{array}$ & $\begin{array}{l}\text { Triangulation } \\
\text { Outcome } \\
\text { Analysis }\end{array}$ & $\begin{array}{l}\text { Participant } \\
\text { assessment (rating } \\
\text { scales, open } \\
\text { ended questions } \\
\text { and focus groups), } \\
\text { Lecturer reflection } \\
\text { and peer } \\
\text { observations }\end{array}$ & $\begin{array}{l}\text { Videos identified as } \\
\text { the most useful and } \\
\text { interesting teaching } \\
\text { tool. Results cross- } \\
\text { validated by lecturer } \\
\text { and peer } \\
\text { observations }\end{array}$ \\
\hline $\begin{array}{l}\text { How video cases } \\
\text { should be used as } \\
\text { authentic stimuli in } \\
\text { problem-based } \\
\text { medical education } \\
\text { [32] }\end{array}$ & $\begin{array}{l}\text { To examine } \\
\text { students views on } \\
\text { the value of video } \\
\text { cases compared to } \\
\text { text based cases. }\end{array}$ & $\begin{array}{l}\text { Medical } \\
\text { Students }\end{array}$ & $\begin{array}{l}\text { Knowledge } \\
\text { Gain }\end{array}$ & $\begin{array}{l}\text { Learner } \\
\text { Reaction } \\
\text { (level 1) }\end{array}$ & Focus Groups & Thematic Analysis & $\begin{array}{l}\text { Video generally } \\
\text { valuable but benefit } \\
\text { dependant on } \\
\text { certain conditions. }\end{array}$ \\
\hline $\begin{array}{l}\text { Visual expertise in } \\
\text { paediatric } \\
\text { neurology [33] }\end{array}$ & $\begin{array}{l}\text { To investigate visual } \\
\text { attention and } \\
\text { cognitive processes } \\
\text { of clinicians of } \\
\text { varying degrees of } \\
\text { experience } \\
\text { diagnosing } \\
\text { authentic paediatric } \\
\text { video case }\end{array}$ & $\begin{array}{l}\text { Medical } \\
\text { Students, Basic } \\
\text { Postgraduate } \\
\text { Training and } \\
\text { Consultant CPD }\end{array}$ & $\begin{array}{l}\text { Knowledge } \\
\text { Gain }\end{array}$ & $\begin{array}{l}\text { Learner } \\
\text { Knowledge } \\
\text { (level 2b) } \\
\text { and } \\
\text { Behaviour } \\
\text { (Level 3) }\end{array}$ & $\begin{array}{l}\text { Observational } \\
\text { study }\end{array}$ & $\begin{array}{l}\text { Eye-tracking data } \\
\text { were analysed } \\
\text { with verbal } \\
\text { recordings. }\end{array}$ & $\begin{array}{l}\text { More experienced } \\
\text { clinicians were } \\
\text { more accurate in } \\
\text { visual diagnosis and } \\
\text { spent more of their } \\
\text { time looking at } \\
\text { relevant areas }\end{array}$ \\
\hline \multirow[t]{2}{*}{$\begin{array}{l}\text { An evaluation of } \\
\text { the effectiveness of } \\
\text { a videotape } \\
\text { programme on } \\
\text { inter-observer } \\
\text { reliability in } \\
\text { outcome } \\
\text { assessment for } \\
\text { osteoarthritis [34] }\end{array}$} & \multirow{2}{*}{$\begin{array}{l}\text { Whether } \\
\text { interobserver } \\
\text { variability in senior } \\
\text { medical students } \\
\text { could be reduced in } \\
\text { a group of patients } \\
\text { with OA using only } \\
\text { a single viewing of } \\
\text { an instructional } \\
\text { videotape. }\end{array}$} & \multirow[t]{2}{*}{$\begin{array}{l}\text { Medical } \\
\text { Students }\end{array}$} & \multirow[t]{2}{*}{$\begin{array}{l}\text { Patient } \\
\text { Examinations } \\
\text { Skills }\end{array}$} & \multirow[t]{2}{*}{$\begin{array}{l}\text { Learner } \\
\text { Behaviour } \\
\text { (level 3) }\end{array}$} & \multirow[t]{2}{*}{$\begin{array}{l}\text { Before and } \\
\text { After Study } \\
\text { [Video } \\
\text { intervention } \\
\text { poorly } \\
\text { described] }\end{array}$} & $\begin{array}{l}\text { Change in mean } \\
\text { values of } \\
\text { previously } \\
\text { described observer } \\
\text { dependant } \\
\text { measures per } \\
\text { participant }\end{array}$ & \multirow{2}{*}{$\begin{array}{l}\text { Pre-standardization } \\
\text { reliability } \\
\text { coefficients were } \\
<0.80 \text { for seven } \\
\text { measures. } \\
\text { Coefficients for the } \\
\text { performance of } \\
\text { knee goniometry } \\
\text { were uniformly low. } \\
\text { Following the } \\
\text { intervention, all but } \\
\text { four reliability } \\
\text { coefficients were } \\
>/=0.93 \text {. }\end{array}$} \\
\hline & & & & & & $\begin{array}{l}\text { Reliability } \\
\text { coeffecients for } \\
\text { the group }\end{array}$ & \\
\hline $\begin{array}{l}\text { An evaluation of } \\
\text { the effectiveness of } \\
\text { a videotape } \\
\text { programme on } \\
\text { inter-observer } \\
\text { reliability in } \\
\text { outcome } \\
\text { assessment for } \\
\text { fibromyalgia [16] }\end{array}$ & $\begin{array}{l}\text { Whether } \\
\text { interobserver } \\
\text { variability in senior } \\
\text { medical students } \\
\text { could be reduced in } \\
\text { a group of patients } \\
\text { with fibromyalgia } \\
\text { using only a single } \\
\text { viewing of an }\end{array}$ & $\begin{array}{l}\text { Medical } \\
\text { Students }\end{array}$ & $\begin{array}{l}\text { Patient } \\
\text { Examinations } \\
\text { Skills }\end{array}$ & $\begin{array}{l}\text { Learner } \\
\text { Behaviour } \\
\text { (level 3) }\end{array}$ & $\begin{array}{l}\text { Before and } \\
\text { After Study } \\
\text { [Video } \\
\text { intervention } \\
\text { poorly } \\
\text { described] }\end{array}$ & $\begin{array}{l}\text { Change in mean } \\
\text { values of } \\
\text { previously } \\
\text { described observer } \\
\text { dependant } \\
\text { measures per } \\
\text { participant }\end{array}$ & $\begin{array}{l}\text { Pre-standardization } \\
\text { reliability } \\
\text { coefficients were } \\
<0.80 \text { for } 8 \\
\text { measures. Following } \\
\text { standardization all } \\
\text { reliability } \\
\text { coefficients, but } \\
\text { one, approximated } \\
\text { or exceeded } 0.80\end{array}$ \\
\hline
\end{tabular}


Table 3 Identification of health care settings in which educational patient video clips have been utilised (Continued)

\begin{tabular}{|c|c|c|c|c|}
\hline & $\begin{array}{l}\text { instructional } \\
\text { videotape. }\end{array}$ & & & \\
\hline $\begin{array}{l}\text { An evaluation of } \\
\text { the effectiveness of } \\
\text { a videotape } \\
\text { programme on } \\
\text { inter-observer } \\
\text { reliability in } \\
\text { outcome } \\
\text { assessment for } \\
\text { ankylosing } \\
\text { spondylitis [18] }\end{array}$ & $\begin{array}{l}\text { Whether } \\
\text { interobserver } \\
\text { variability in senior } \\
\text { medical students } \\
\text { could be reduced in } \\
\text { a group of patients } \\
\text { with ankylosing } \\
\text { spondylitis using } \\
\text { only a single } \\
\text { viewing of an } \\
\text { instructional } \\
\text { videotape. }\end{array}$ & $\begin{array}{l}\text { Medical } \\
\text { Students }\end{array}$ & $\begin{array}{l}\text { Patient } \\
\text { Examinations } \\
\text { Skills }\end{array}$ & $\begin{array}{l}\text { Learner } \\
\text { Behaviour } \\
\text { (level 3) }\end{array}$ \\
\hline
\end{tabular}

Reliability
coeffecients for
the group

After Study

Change in mean

Pre-standardization

Video previously

coefficients were <

intervention

described observer 0.80 for three

poorly

dependant

described]

measures per

participant

measures. Following

Reliability

coeffecients for

standardization 12

reliability

the group

coefficients

exceeded 0.80. For

the majority of

measures pre-

standardization

reliability

coefficients were

high and no further improvement in

reliability could be

demonstrated

\begin{tabular}{|c|c|c|c|c|c|c|c|}
\hline \multirow{2}{*}{$\begin{array}{l}\text { An evaluation of } \\
\text { the effectiveness of } \\
\text { a videotape } \\
\text { programme on } \\
\text { inter-observer } \\
\text { reliability in } \\
\text { outcome } \\
\text { assessment for } \\
\text { rheumatoid arthritis } \\
{[17]}\end{array}$} & \multirow{2}{*}{$\begin{array}{l}\text { Whether } \\
\text { interobserver } \\
\text { variability in senior } \\
\text { medical students } \\
\text { could be reduced in } \\
\text { a group of patients } \\
\text { with rheumatoid } \\
\text { arthritis using only a } \\
\text { single viewing of an } \\
\text { instructional } \\
\text { videotape. }\end{array}$} & \multirow[t]{2}{*}{$\begin{array}{l}\text { Medical } \\
\text { Students }\end{array}$} & \multirow[t]{2}{*}{$\begin{array}{l}\text { Patient } \\
\text { Examinations } \\
\text { Skills }\end{array}$} & \multirow[t]{2}{*}{$\begin{array}{l}\text { Learner } \\
\text { Behaviour } \\
\text { (level 3) }\end{array}$} & \multirow[t]{2}{*}{$\begin{array}{l}\text { Before and } \\
\text { After Study } \\
\text { [Video } \\
\text { intervention } \\
\text { poorly } \\
\text { described] }\end{array}$} & $\begin{array}{l}\text { Change in mean } \\
\text { values of } \\
\text { previously } \\
\text { described observer } \\
\text { dependant } \\
\text { measures per } \\
\text { participant }\end{array}$ & \multirow{2}{*}{$\begin{array}{l}\text { Pre-standardization } \\
\text { reliability } \\
\text { coefficients were } \\
>0.80 \text { for all } \\
\text { measures and } \\
\text { remained above } \\
0.80 \text { following } \\
\text { standardization } \\
\text { except for one } \\
\text { measure }\end{array}$} \\
\hline & & & & & & $\begin{array}{l}\text { Reliability } \\
\text { coeffecients for } \\
\text { the group }\end{array}$ & \\
\hline \multirow{2}{*}{$\begin{array}{l}\text { Osteoarthritis } \\
\text { antirheumatic drug } \\
\text { trials: Effects of a } \\
\text { standardized } \\
\text { instructional } \\
\text { videotape on the } \\
\text { reliability of } \\
\text { observer-dependent } \\
\text { dependent } \\
\text { outcome measures } \\
\text { [35] }\end{array}$} & \multirow{2}{*}{$\begin{array}{l}\text { Whether } \\
\text { interobserver } \\
\text { variability in } \\
\text { consultants could } \\
\text { be reduced in a } \\
\text { group of patients } \\
\text { with OA using only } \\
\text { a single viewing of } \\
\text { an instructional } \\
\text { videotape. }\end{array}$} & \multirow[t]{2}{*}{ Consultant CPD } & \multirow[t]{2}{*}{$\begin{array}{l}\text { Patient } \\
\text { Examination } \\
\text { Skills }\end{array}$} & \multirow[t]{2}{*}{$\begin{array}{l}\text { Learner } \\
\text { Behaviour } \\
\text { (level 3) }\end{array}$} & \multirow[t]{2}{*}{$\begin{array}{l}\text { Before and } \\
\text { After Study } \\
\text { [Video } \\
\text { intervention } \\
\text { poorly } \\
\text { described] }\end{array}$} & $\begin{array}{l}\text { Change in mean } \\
\text { values of } \\
\text { previously } \\
\text { described observer } \\
\text { dependant } \\
\text { measures per } \\
\text { participant }\end{array}$ & \multirow[t]{2}{*}{$\begin{array}{l}\text { Prestandardization } \\
\text { reliability } \\
\text { coefficients were } \\
>0.80 \text { for all } \\
\text { measures and } \\
\text { remained above } \\
0.80 \text { following the } \\
\text { intervention }\end{array}$} \\
\hline & & & & & & $\begin{array}{l}\text { Reliability } \\
\text { coeffecients for } \\
\text { the group }\end{array}$ & \\
\hline \multirow{2}{*}{$\begin{array}{l}\text { Fibromyalgia } \\
\text { antirheumatic drug } \\
\text { trials: Effects of a } \\
\text { standardized } \\
\text { instructional } \\
\text { videotape on the } \\
\text { reliability of } \\
\text { observer-dependent } \\
\text { outcome measures } \\
\text { [19] }\end{array}$} & \multirow{2}{*}{$\begin{array}{l}\text { Whether } \\
\text { interobserver } \\
\text { variability in } \\
\text { consultants could } \\
\text { be reduced in a } \\
\text { group of patients } \\
\text { with fibromyalgia } \\
\text { using only a single } \\
\text { viewing of an } \\
\text { instructional } \\
\text { videotape. }\end{array}$} & \multirow[t]{2}{*}{ Consultant CPD } & \multirow[t]{2}{*}{$\begin{array}{l}\text { Patient } \\
\text { Examinations } \\
\text { Skills }\end{array}$} & \multirow[t]{2}{*}{$\begin{array}{l}\text { Learner } \\
\text { Behaviour } \\
\text { (level 3) }\end{array}$} & \multirow[t]{2}{*}{$\begin{array}{l}\text { Before and } \\
\text { After Study } \\
\text { [Video } \\
\text { intervention } \\
\text { poorly } \\
\text { described] }\end{array}$} & $\begin{array}{l}\text { Change in mean } \\
\text { values of } \\
\text { previously } \\
\text { described observer } \\
\text { dependant } \\
\text { measures per } \\
\text { participant }\end{array}$ & \multirow{2}{*}{$\begin{array}{l}\text { Prestandardization } \\
\text { reliability } \\
\text { coefficients were } \\
<0.80 \text { for } 8 \\
\text { measures. Following } \\
\text { standardization all } \\
\text { reliability } \\
\text { coefficients } \\
\text { approximated to or } \\
\text { exceeded } 0.80 \text {. }\end{array}$} \\
\hline & & & & & & $\begin{array}{l}\text { Reliability } \\
\text { coeffecients for } \\
\text { the group }\end{array}$ & \\
\hline \multirow{2}{*}{$\begin{array}{l}\text { Rheumatoid arthritis } \\
\text { antirheumatic drug } \\
\text { trials: Effects of a } \\
\text { standardized } \\
\text { instructional } \\
\text { videotape on the } \\
\text { reliability of } \\
\text { observer-dependent } \\
\text { outcome measures } \\
\text { [20] }\end{array}$} & \multirow{2}{*}{$\begin{array}{l}\text { Whether } \\
\text { interobserver } \\
\text { variability in } \\
\text { consultants could } \\
\text { be reduced in a } \\
\text { group of patients } \\
\text { with rheumatoid } \\
\text { arthritis using only a } \\
\text { single viewing of an } \\
\text { instructional } \\
\text { videotape. }\end{array}$} & \multirow[t]{2}{*}{ Consultant CPD } & \multirow[t]{2}{*}{$\begin{array}{l}\text { Patient } \\
\text { Examinations } \\
\text { Skills }\end{array}$} & \multirow[t]{2}{*}{$\begin{array}{l}\text { Learner } \\
\text { Behaviour } \\
\text { (level 3) }\end{array}$} & \multirow[t]{2}{*}{$\begin{array}{l}\text { Before and } \\
\text { After Study } \\
\text { [Video } \\
\text { intervention } \\
\text { poorly } \\
\text { described] }\end{array}$} & $\begin{array}{l}\text { Change in mean } \\
\text { values of } \\
\text { previously } \\
\text { described observer } \\
\text { dependant } \\
\text { measures per } \\
\text { participant }\end{array}$ & \multirow[t]{2}{*}{$\begin{array}{l}\text { Prestandardization } \\
\text { reliability } \\
\text { coefficients were } \\
>0.80 \text { for all } \\
\text { measures and } \\
\text { remained above } \\
0.80 \text { following } \\
\text { standardization }\end{array}$} \\
\hline & & & & & & $\begin{array}{l}\text { Reliability } \\
\text { coeffecients for } \\
\text { the group }\end{array}$ & \\
\hline
\end{tabular}


Table 3 Identification of health care settings in which educational patient video clips have been utilised (Continued)

\begin{tabular}{|c|c|c|c|c|c|c|}
\hline \multirow{2}{*}{$\begin{array}{l}\text { Ankylosing } \\
\text { spondylitis } \\
\text { antirheumatic drug } \\
\text { trials: Effects of a } \\
\text { standardized } \\
\text { instructional } \\
\text { viddeotape on the } \\
\text { reliability of } \\
\text { observer-dependent } \\
\text { outcome measures } \\
\text { [21] }\end{array}$} & \multirow{2}{*}{$\begin{array}{l}\text { Whether } \\
\text { interobserver } \\
\text { variability in } \\
\text { consultants could } \\
\text { be reduced in a } \\
\text { group of patients } \\
\text { with ankylosing } \\
\text { spondylitis using } \\
\text { only a single } \\
\text { viewing of an } \\
\text { instructional } \\
\text { videotape. }\end{array}$} & \multirow[t]{2}{*}{$\begin{array}{l}\text { Patient } \\
\text { Examinations } \\
\text { Skills }\end{array}$} & \multirow[t]{2}{*}{$\begin{array}{l}\text { Learner } \\
\text { Behaviour } \\
\text { (level 3) }\end{array}$} & \multirow[t]{2}{*}{$\begin{array}{l}\text { Before and } \\
\text { After Study } \\
\text { [Video } \\
\text { intervention } \\
\text { poorly } \\
\text { described] }\end{array}$} & $\begin{array}{l}\text { Change in mean } \\
\text { values of } \\
\text { previously } \\
\text { described observer } \\
\text { dependant } \\
\text { measures per } \\
\text { participant }\end{array}$ & \multirow{2}{*}{$\begin{array}{l}\text { Prestandardization } \\
\text { reliability } \\
\text { coefficients were } \\
<0.80 \text { for three } \\
\text { measures. Following } \\
\text { standardization } 12 \\
\text { reliability } \\
\text { coefficients } \\
\text { exceeded } 0.80\end{array}$} \\
\hline & & & & & $\begin{array}{l}\text { Reliability } \\
\text { coeffecients for } \\
\text { the group }\end{array}$ & \\
\hline
\end{tabular}

trained doctors continuing professional development. Noting the reasons given above the total number of articles evaluated was 17 (which involved 18 distinct studies).

Table 3 contains the descriptive results for the reviewed articles and Table 4 contains the overall judgement on each of the articles. The analysis of the validity of the outcome measures can be found in the Additional file 1: Appendix.

\section{Discussion}

This review examined the evidence on how to measure outcomes when Patient Video Cases (PVCs) are used in healthcare settings. This evidence was small, extremely heterogeneous and there was insufficient evidence to specify the best outcomes to use. The heterogeneity in the articles was created by the diversity of involved health care professionals, varying educational purposes, different types of intervention, a wide range of outcome methodologies, different internal and construct validities and a variety of results. Each of these is examined in turn.

\section{Type of healthcare professional}

The preponderance of projects in undergraduate education is likely related to the large number of medical education academics at these institutions, the access to a 'captive group' of subjects and the greater ease of assessing undergraduate outcomes. Further investigation into the use of PVCs at postgraduate level and in other healthcare professionals is clearly warranted. For all health care professionals it is also reasonable to attribute the lack of studies to the difficulties in designing [36] and funding studies evaluating PVCs.

\section{Educational purposes and types of intervention}

Given the small number of studies, it is difficult to identify clear treads in educational purpose or type of intervention. Learner satisfaction and knowledge gain are the easiest of the Kirkpatrick training outcomes to measure as they do not require external observation or intervention. However these domains are the lowest in the hierarchy of evidence needed to confirm that a training process has been truly effective [37]. No study looked at organisational change, which is in keeping with previous literature. A review aiming to identify methods used to measure change in the clinical practices of health professionals found only $17.6 \%$ looked at changes at an organisation level [38]. Also in this review only one study attempted to look at more than one level of training outcome. A systematic review of evaluation in formal continuing medical education [39] noted $28 \%$ of studies reviewed looked at two levels and only 6\% looked at three.

\section{Methods for determining and assessing outcome measures}

Reflecting the wide range of different types of studies performed, the validity of the outcome measures used was variable. This represents the difficulties of examining interventions related to education and training. In clinical practice a gold standard approach in assessing the effectiveness of medication is the randomised controlled trial. The primary outcome measure being an objective endpoint such as a defined reduction or gain in a physiological parameter. In training interventions, a single endpoint as an outcome requires a lot of interpretation, and potential criticism. For example, learner satisfaction does not necessarily equate to knowledge change, neither does it have a direct correlation with change in practice. The absence of a gold standard measure to assess training interventions may have led researchers to be opportunistic in their use of outcome measures. In this review seven studies gave no justification for the outcome measure used [13,15,25,26,29,30]. In addition comments by the authors themselves on limitations to the outcome measures were absent in five of the studies [13,26,30,31].

Only one study looked at more than one discrete domain in the Kirkpatrick training evaluation framework [29]. In this work both learner knowledge and learner satisfaction were assessed by different measures (a video test, a written test and a course evaluation). Three other studies $[14,25,31]$ had more than one outcome measure, although these were all subtle variations on a theme 
Table 4 Review of methodological quality of studies using outcome measures to assess the impact of PVCs

\begin{tabular}{|c|c|c|}
\hline Paper & 1 & 2 \\
\hline & $\begin{array}{l}\text { How is the choice of } \\
\text { outcome measure } \\
\text { justified? }\end{array}$ & $\begin{array}{l}\text { Will this choice } \\
\text { determine the results } \\
\text { the study aims to } \\
\text { investigate? }\end{array}$ \\
\hline
\end{tabular}

34

To what extent are How do they

$\overline{\text { Internal }}$ Construct

the writers aware of overcome the validity? validity?

the disadvantages as disadvantages?

well as the

advantages of the

outcome measures chosen?

Using interactive video Used to justify criterion Study aims not clear. If

to add physical validity of the

assessment data to intervention used.

computer based

patient simulations [22] presumed to be to

elucidate whether the

simulations are an

effective learning

experience the

outcome used partially

confirms the simulation

represent normal

practice not that the

simulation improved performance or was an useful education tool

The development of shared cognition in paediatric residents analysing a patient video versus a paper patient case [23]

\section{Yes}

Process of capturing concept link formation described with the reason for using verbal protocol analysis supported by published evidence.

Methodology of using simulated recall in individuals following the group discussions not well supported.
Enhancing diagnostic accuracy among nonexperts through use of video cases [24]
Previous work by the author has shown improved cognitive processes when PVCs utilised. Improved diagnostic accuracy is the natural conclusion of relevant or improved cognitive processing.

(1)

Yes

Yes

No justification for answer analysis is given although video clips into computer-based testing: Effects on item statistics and reliability estimates [25]

\section{Video-based test} questions: A novel means of evaluation [26]

No justification for questionnaire methodology given although this format is

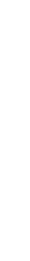

Author notes that in actual practice chart noting is done under time pressure whereas with this simulation there was more time available to make case-notes more complete.

$$
\text { complete. }
$$

No comment made on No No
this. The absence of
details on whether a
specific proforma for
extracting information
from the case-notes
was used makes it
difficult to assess how
comparisons were
made.

No (1)

Authors note a small number of participants and only one case so reliability may be questioned.

They also note the ability to accurately recall and record all concept links is not established.

They were aware the interviewers were not blinded to the intervention group of the participants.

Study acknowledges the diagnostic accuracy as an outcome is only a short term variable of learning.

Authors touch on, but don't specifically note, the outcome measure is not directly related to the intervention rather the group discussion following the intervention.

Problems with questions with low discrimination values identified and subject to supplemental analysis. an accepted primary approach to gathering information on satisfaction with a process.
The positioning of the simulated recall exercise straight after the group work limits loss of content due to degradation of memories.

Authors comment although not blinded the interviewers were not recording cognitive processes lead to them.

Authors argue increase in diagnostic reasoning in non-experts in may promote further literature study and learning.

$\begin{array}{lll}\begin{array}{l}\text { Items with RPB values } \\ \text { of zero or less than 0.2 }\end{array} & \text { Applicable } & \text { Applicable } \\ \text { removed although no } & & \\ \begin{array}{l}\text { explanation of why } \\ \text { these values were }\end{array} & & \\ \text { chosen. } & & \\ \text { No comments made } & \text { Not } & \text { Not } \\ & \text { Applicable } & \text { Applicable }\end{array}$

Yes Yes

Yes

Yes just the thoughts that
Yes although reliability No comments made of results must be treated with caution. 
Table 4 Review of methodological quality of studies using outcome measures to assess the impact of PVCs (Continued)

\begin{tabular}{|c|c|}
\hline $\begin{array}{l}\text { Video-based test } \\
\text { questions: A novel } \\
\text { means of evaluation } \\
{[26]}\end{array}$ & No justification given \\
\hline $\begin{array}{l}\text { A comparison of } \\
\text { critical thinking in } \\
\text { groups of third-year } \\
\text { medical students in } \\
\text { text, video, and virtual } \\
\text { PBL case modalities } \\
{[27]}\end{array}$ & $\begin{array}{l}\text { There is a theoretical } \\
\text { association between } \\
\text { Problem Based } \\
\text { Learning and critical } \\
\text { thinking. A sound } \\
\text { research framework } \\
\text { exists to analyse } \\
\text { discourse and code for } \\
\text { content. Therefore a } \\
\text { process, discourse } \\
\text { analysis, exists to } \\
\text { examine the outcomes } \\
\text { of PBL in respect to } \\
\text { critical thinking. }\end{array}$ \\
\hline
\end{tabular}

Comparison of text and video cases in a postgraduate problembased learning format [28]

Use of animationenhanced video clips for teaching abnormal breathing patterns [13]

\begin{abstract}
Using web-based video to enhance physical examination skills in medical students [14]
\end{abstract}

Teaching the plantar reflex [15]

A videotape-based training method for improving the detection of depression in residents cognitive and

metacognitive thinking has been established.

It is theoretically plausible a video case would improve thinking processes. for questionnaire methodology although this format is an accepted primary approach to gathering information on satisfaction with a process. skills assessment has been utilised (and presumably validated although this is not stated) in the learning institution the study is taking place in.

No justification given. Process for assessing performance described although the standardised rating scale was not demonstrated.

Outcome measures well described but no comment on the reason for using them.
A coding system for

No justification given

Summative clinical Aims of the study not No comments made
clear

No comments made

Yes depends on the

Yes. Although the actual reason for improved educational outcome in PBL has yet to be defined and the coding schema chosen is only one way of evaluating cognitive and metacognitive processes.

Yes although the reliability of results must be treated with caution.

Yes, although dependant on the reliability of the Clinical Skills Assessment.

utilisation of the video clip website by

Yes but only if the assessment system is valid.

As patient outcome not measured methodology can only assess how the training programme improves performance validity and reliability of the coder.

Although the coder is blind to group type it is possible for this to be suggested by the dialogue.

Time pressures on face-to-face groups may limit opportunities to refine critical thinking compared to the virtual groups.

The outcome measure itself is not examined although the article notes the use of one author for both groups and the low numbers of residents in both groups mean caution is required in interpretation.

No comment made. The authors note that some respondents gave inconsistent written comments when compared with their agreement with statements and comment it was likely they had misunderstood the question.

The authors comment they did not track the students and note a prospective, randomised controlled study would have been more accurate.

The authors

acknowledge the effect of the entrance test in providing education in itself.

Only one author did all Yes

Yes the coding but coding agreement was checked using a sample of transcripts with two others (one not involved in the study).

The use of clause frequency enables variability in group sizes to be addressed.

No comments made

Potentially Yes Not

Authors not a large sample size is needed

Authors tested after a control period and

Potentially Yes withheld feedback to participants about their attrition. test performances.
Potentially Yes Not 
Table 4 Review of methodological quality of studies using outcome measures to assess the impact of PVCs (Continued)

of long-term care
facilities [29]

Advantages of video trigger in problembase learning [30]

A triangulated approach to the assessment of teaching in childhood epilepsy [31]

How video cases should be used as authentic stimuli in problem-based medical education [32]

Visual expertise in paediatric neurology [33]

An evaluation of the effectiveness of a videotape programme on inter-observer reliability in outcome assessment for osteoarthritis [34]

Osteoarthritis antirheumatic drug trials: Effects of a standardized instructional videotape on the reliability of observer-dependent dependent outcome measures [35] in the outcomes tested.

No justification for questionnaire methodology given although this format is an accepted primary approach to gathering information on satisfaction with a process.

Triangulation used as a more complete and robust measure to validate findings. employed in a methodological
Yes although must be fashion. However the use of video clips was not the sole purpose of the study and questions not posed to determine this.

Focus groups a well refined qualitative tool which all deep analysis of concepts presented. Yes

Important differences Yes in perception between experts and novices studying dynamic stimuli has been documented. Authors note this field is underexplored in the medical domain but use a high quality eye tracking machine and linked to spoken cognitive processes

Outcome measure used in previous studies to assess performance in musculo-skeletal examination.

Outcome measure Yes used in previous studies to assess performance in musculo-skeletal examination.
They also not the

vignette video test

may improve practice

in its own right.

Staff were allowed to

choose timing and

type of session

according to their

needs with no control

for group or individual sessions.

Yes as long as sample No comments made of participants valid.

Very little attention paid to confounding influences and the fact

No comments made No

Not that the cross -

validation was not particular well demonstrated.

Clear

acknowledgement of

Very experienced

facilitator used

Yes

Yes the problems with individuals dominating or evading group discussion.

Note that outcome method was novel and made efforts to triangulate findings to gold standard outcomes (such as correct diagnosis)

Used variety of experience in subject population
A larger matrix and more observers and patients may have been used to improve reliability.

A larger matrix and more observers and patients may have been used to improve reliability.
The authors own previous work has indicated the $6 \times 6$ is pragmatic and representative.

The authors own previous work has indicated the $6 \times 6$ is pragmatic and representative. 
such as scores in different types of clinical examination in the same test.

Only two of the studies [27,33] satisfied all domains when deciding on whether internal and construct validity had been achieved. Three other papers [15,23,29] had minor concerns, generally relating to the extent which the outcome measure itself affected the results. Questionnaire studies reflecting learner satisfaction tended not to perform well as control between groups was not possible and confounding factors were very difficult to assess.

\section{Results of the interventions}

Nearly all papers were positive regarding the use of PVCs (regardless of whether the analysis above had revealed concerns over the validity of the outcome measure). The medical student studies regarding critical analysis and thinking showed strong results in favour of the use of PVCs. The underlying hypotheses of these studies $[23,24,27,28,32]$ were plausible and the methodologies used rigorous. A researcher independent of these groups has also recently shown students prefer this use of PVCs to current problem based learning techniques [30] so triangulation has in some respects been achieved in this field. A recent paper demonstrating experts are more focused on the relevant clinical features within patient video clips has been further supported by, as yet unpublished evidence, that eye movement modelling may improve diagnostic reasoning. This methodology, where the minute movements of the eye are tracked while observing dynamic images, has strong construct validity. It is felt the cognitive 'load' of dynamic video clips may encourage cognitive processing [40] and therefore methodologies to explore the extent of this load created by PVCs are welcome. Future research must be cognisant of the fact that under- or over-load may occur depending on the capacity of the individual engaging in the activity. Extraneous cognitive [41] load may be able to be controlled to some extent by investigators and this will aid determination of its impact on the outcome of the intervention.

Studies concerning testing methods and clinical examination showed no obvious differences between PVCs and current assessment methods. The potential difficulty and cost of placing video clips into examinations (whether formative or summative) may have limited the number of validation studies in this area. In studies of clinical examination technique which aimed to show improvement following a PVC intervention, there was supportive evidence although initial skill sets tended to be relatively high. The importance of controlling for this was demonstrated by the use of the Solomon Four Group design on a video intervention to improve examination of the plantar reflex [15]. In this study an effect was only seen when pre-intervention performance was assessed.
The video-based training method for improving the detection of depression in residents of long term care facilities demonstrated an increase in performance of the intervention group in both knowledge assessments [29]. Direct patient benefit was not assessed so an improvement in clinical care as a use of PVC cannot be claimed. However given the good levels of satisfaction on questionnaire testing it is likely that participants would not have been averse to incorporating newly acquired learning into their day-to-day practice.

\section{Limitations}

The heterogeneity of the current published evidence made a robust narrative review extremely difficult. Apart from the work on how PVCs encourage discourse and critical thinking, there were no common themes in which to be able to extract information and analyse composite outcomes. This may represent difficulty in undertaking research in the field (the cost of production of video clips), the difficulty in defining valid outcome measures or publication bias due to a paucity of positive outcomes. This exemplifies the challenge that much medical education research is Action Research, research based on the instructors' own practice.

Publication bias is unlikely to be significant as there as there is literature in which research is positive [42] regarding the use of video and online technologies but there are also negative [43] publications in existence. It would seem unlikely a particular modality of online or audiovisual learning would be subject to a different research agenda.

The main limitation of this study is the low number of articles that were found. The search strategy used was expansive although "Patient Video Clip" or similar terms are not used by all researchers in the field. It is possible terms other than those searched have been used although the number of papers missed is likely to be very small. Extraction of data was performed by a sole reviewer so it is possible so errors of typology were made although the small number of final articles has allowed extensive examination of the papers by all the authors.

\section{Conclusion}

This review process has demonstrated the diverse nature of research in determining the effectiveness of PVCs in education. Medical education occurs in a variety of environments and the complicated interplay of confounding variables makes interpretation of outcomes difficult. The following recommendations would enable the production of a standard conceptual framework to guide future research in the area.

- Studies should classify which facet of training or educational outcome the study is aiming to explore. 
- Studies should aim to validate a particular outcome measure, preferably by reproducing previous work rather than adopting new methods.

- A description of the validity of the chosen outcome measure should be included in study protocol.

- Although control groups are useful for demonstrating the benefit of a PVC intervention, more evidence is needed on whether the outcome measure demonstrates construct validity.

- Studies on PVCs should take account of cognitive theory with the cognitive processing enhancement, demonstrated in a number of the medical student papers, tested at a postgraduate level. Although pragmatic outcome measures are easier to achieve explanatory trials are needed.

- Prior-knowledge and behaviour testing is vital to demonstrate improvement.

\section{Additional file}

Additional file 1: Appendix. Review of studies to assist in evaluation of internal and construct validity based on framework via Farringdon.

\section{Competing interests}

The author(s) declare that they have no competing interests.

This report is independent research arising from a Doctoral Research Fellowship supported by the National Institute for Health Research. The views expressed in this publication are those of the author(s) and not necessarily those of the NHS, the National Institute for Health Research or the Department of Health.

\section{Authors' contributions}

DR proposed the original research concept, carried out the literature review and prepared a first draft. DM and TC made significant contributions to the design and methodology of the review as well as to subsequent drafting of the article. All authors read and approved the final manuscript.

\section{Acknowledgements}

The authors wish to acknowledge the help given by Sarah Sutton, senior medical librarian at the University Hospitals of Leicester NHS Trust, in the preparation of the literature search.

\section{Author details}

Emergency Medicine Academic Group, Emergency Department secretaries c/o Elizabeth Cadman-Moore, Leicester Royal Infirmary, Leicester LE1 5WW, UK. ${ }^{2}$ Room B94C Medical School, Queens Medical Centre, Nottingham NG7 $2 \mathrm{UH}, \mathrm{UK}$.

Received: 30 March 2012 Accepted: 12 December 2012

Published: 21 December 2012

\section{References}

1. McNulty JA, Hoyt A, Gruener G, Chandrasekhar A, Espiritu B, Price R Jr, Naheedy R: An analysis of lecture video utilization in undergraduate medical education: associations with performance in the courses. BMC Med Educ 2009, 9:6.

2. Leone TA, Rich W, Finer NN: Educational Perspectives. NeoReviews 2006, 7(12):e597-e601.

3. Romanov K, Nevgi A: Do medical students watch video clips in eLearning and do these facilitate learning? Med Teach 2007, 29(5):484-488.

4. Roland D, Wahl H, Lakhanpaul M, Blackwell N, Davies F: Education by video: BMJ (Careers); 2011. available at http://careers.bmj.com/careers/advice/viewarticle.html?id=20001865 (website last accessed $14^{\text {th }}$ December 2012).

5. (National Patient Safety Agency) NPSA: Review of patient safety for children and young people:; 2009. available via http://www.nrls.npsa.nhs.uk/EasySiteWeb/
getresource.axd?AssetID=60227\&type=full\&servicetype=Attachment (website last accessed $14^{\text {th }}$ December 2012).

6. Bates R: A critical analysis of evaluation practice: the Kirkpatrick model and the principle of beneficence. Eval Program Plann 2004, 27(3):341-347.

7. Kirkpatrick D, Kirkpatrick J: Evaluating Training Programs: The Four Levels: $3^{\text {rd }}$ ed. San Francisco: Berrett-Koehler Publishers, Inc.; 2006.

8. $\quad$ Arthur W Jr, Bennett W Jr, Edens PS, Bell ST: Effectiveness of training in organizations: a meta-analysis of design and evaluation features. J Appl Psychol 2003, 88(2):234-245.

9. Barr H, Freeth D, Hammick M, Koppel I, Reeves S: Evaluations of interprofessional education: a United Kingdom review for health and social care. 1st edition. London: London: Centre for the Advancement of Interprofessional Education; 2000.

10. Cook T, Campbell D: Quasi-experimentation: Design and analysis issues for field settings: First ed. Boston, MA: Houghton Mifflin Company; 1979.

11. Shadish W, Cook T, Campbell D: Experimental and quasi-experimental designs for generalized causal inference: First ed. Boston, MA: Houghton Mifflan Company; 2002.

12. Farrington DP: Methodological Quality Standards for Evaluation Research. Ann Am Acad Pol Soc Sci 2003, 587(1):49-68.

13. Hawkins EC, Hansen B, Bunch BL: Use of animation-enhanced video clips for teaching abnormal breathing patterns. J Vet Med Educ 2003, 30(1):73-77.

14. Orientale E Jr, Kosowicz L, Alerte A, Pfeiffer C, Harrington K, Palley J, Brown S, Sapieha-Yanchak T: Using web-based video to enhance physical examination skills in medical students. Fam Med 2008, 40(7):471-476.

15. Raijmakers PG, Cabezas MC, Smal JA, van Gijn J: Teaching the plantar reflex. Clin Neurol Neurosurg 1991, 93(3):201-204.

16. Bellamy N, Anjema C, Chhina T, Dudek N, Hurley B, Landesman B, Probyn L, Hill J, Campbell J: An evaluation of the effectiveness of a videotape programme on interobserver reliability in outcome assessment for fibromyalgia. Inflammopharmacology 1999, 7(2):119-130.

17. Bellamy N, Anjema C, Frankovic TS, Horowitz N, Mills GCR, Shulman S, Smith C, Hill J, Campbell J: An evaluation of the effectiveness of a videotape programme on interobserver reliability in outcome assessment for rheumatoid arthritis. Inflammopharmacology 1999, 7(2):131-141.

18. Bellamy N, Anjema C, Dhanoa D, Joglekar A, Mills GC, Nesrallah G, Smith C, Ucar C, Hill J, Campbell J: An evaluation of the effectiveness of a videotape programme on interobserver reliability in outcome assessment for ankylosing spondylitis. Inflammopharmacology 1999, 7(2):107-117.

19. Bellamy N, Buchbinder R, Hall S, Lowthian P, Patrick M, Ryan P, Stockman A, Grant G, Green S, Campbell J: Fibromyalgia antirheumatic drug trials: Effects of a standardized instructional videotape on the reliability of observer-dependent outcome measures. Inflammopharmacology 1997, 5(3):261-272.

20. Bellamy N, Muirden K, Bendrups A, Boyden K, McColl G, Moran H, Stockman A, Tellus M, Travers R, Campbell J: Rheumatoid arthritis antirheumatic drug trials: Effects of a standardized instructional videotape on the reliability of observer-dependent outcome measures. Inflammopharmacology 1997, 5(3):273-284.

21. Bellamy N, Muirden K, Boyden K, McColl G, Moran H, Stockman A, Tellus M, Wicks I, Campbell J: Ankylosing spondylitis antirheumatic drug trials: Effects of a standardized instructional viddeotape on the reliability of observerdependent outcome measures. Inflammopharmacology 1997, 5(3):297-308.

22. White JE: Using interactive video to add physical assessment data to computer-based patient simulations in nursing. Comput Nurs 1995, 13(5):233-235.

23. Balslev T, de Grave W, Muijtjens AMM, Eika B, Scherpbier AJJA: The development of shared cognition in paediatric residents analysing a patient video versus a paper patient case. Adv Health Sci Educ 2009, 14(4):557-565.

24. Balslev T, De Grave WS, Muijtjens AMM, Scherpbier AJJA: Enhancing diagnostic accuracy among nonexperts through use of video cases. Pediatrics 2010, 125(3).

25. Lieberman SA, Frye AW, Litwins SD, Rasmusson KA, Boulet JR: Introduction of patient video clips into computer-based testing: Effects on item statistics and reliability estimates. Acad Med 2003, 78(10 SUPPL).

26. Hertenstein MJ, Wayand JF: Video-based test questions: A novel means of evaluation. J Instr Psychol 2008, 35(2):188-191.

27. Kamin C, O'Sullivan P, Deterding R, Younger M: A comparison of critical thinking in groups of third-year medical students in text, video, and virtual PBL case modalities. Acad Med 2003, 78(2):204-211. 
28. Balslev T, de Grave WS, Muijtiens AM, Scherpbier AJ: Comparison of text and video cases in a postgraduate problem-based learning format. Med Educ 2005, 39(11):1086-1092.

29. Wood S, Cummings IL, Schnelle B, Stephens M: A videotape-based training method for improving the detection of depression in residents of long-term care facilities. Gerontologist 2002, 42(1):114-121.

30. Chan LK, Patil NG, Chen JY, Lam JC, Lau CS, Ip MS: Advantages of video trigger in problem-based learning. Med Teach 2010, 32(9):760-765.

31. Bye AM, Connolly AM, Netherton C, Looker P, Burgess A, Lonergan A: A triangulated approach to the assessment of teaching in childhood epilepsy. Med Teach 2007, 29(2-3):255-257.

32. de Leng B, Dolmans $D$, van de Wiel M, Muijtjens $A$, van der Vleuten $C$ : How video cases should be used as authentic stimuli in problembased medical education. Med Educ 2007, 41(2):181-188.

33. Balslev T, Jarodzka H, Holmqvist K, de Grave W, Muijtjens AM, Eika B, van Merrienboer J, Scherpbier AJ: Visual expertise in paediatric neurology. Eur J Paediatr Neurol 2012, 16(2):161-166.

34. Bellamy N, Anjema C, Alikhan N, Chhina T, Dhanoa D, Edelist D, Esufali Z, Ismail F, Hill J, Campbell J: An evaluation of the effectiveness of a videotape programme on interobserver reliability in outcome assessment for osteoarthritis. Inflammopharmacology 1999, 7(2):143-154.

35. Bellamy N, Bachmeier C, Brooks P, Browne C, Cohen M, March L, Conaghan P, Day R, Campbell J: Osteoarthritis antirheumatic drug trials: Effects of a standardized instructional videotape on the reliability of observer-dependent dependent outcome measures. Inflammopharmacology 1997, 5(3):285-295.

36. Norman G: RCT = results confounded and trivial: the perils of grand educational experiments. Med Educ 2003, 37(7):582-584.

37. Kirkpatrick D: Evaluation of training. In Training and development handbook: A guide to human resource development. 1st edition. Edited by Craig RL. New York: McGraw-Hill; 1976:317.

38. Hakkennes $\mathrm{S}$, Green $\mathrm{S}$ : Measures for assessing practice change in medical practitioners. Implement Sci 2006, 1:29.

39. Tian J, Atkinson N, Portnoy B, Gold R: A systematic review of evaluation in formal continuing medical education. J Contin Educ Health Prof 2007, 27(1):16-27.

40. Paas F, Renkl A, Sweller J: Cognitive Load Theory and Instructional Design: Recent Developments. Educ Psychol 2003, 38(1):1-4.

41. Chandler P, Sweller J: Cognitive Load Theory and the Format of Instruction. Cognit Instruct 1991, 8(4):293-332.

42. Hadley J, Kulier R, Zamora J, Coppus SF, Weinbrenner S, Meyerrose B, Decsi T, Horvath AR, Nagy E, Emparanza Jl, Arvanitis TN, Burls A, Cabello JB, Kaczor M, Zanrei G, Pierer K, Kunz R, Wilkie V, Wall D, Mol BW, Khan KS: Effectiveness of an e-learning course in evidence-based medicine for foundation (internship) training. J R Soc Med 2010, 103(7):288-294.

43. Bakker RJ, van de Putte EM, Kuis W, Sinnema G: Effects of an educational video film in fatigued children and adolescents: a randomised controlled trial. Arch Dis Child 2011, 96(5):457-460.

doi:10.1186/1472-6920-12-125

Cite this article as: Roland et al:: Towards a conceptual framework demonstrating the effectiveness of audiovisual patient descriptions (patient video cases): a review of the current literature. BMC Medical Education 2012 12:125.

\section{Submit your next manuscript to BioMed Central and take full advantage of:}

- Convenient online submission

- Thorough peer review

- No space constraints or color figure charges

- Immediate publication on acceptance

- Inclusion in PubMed, CAS, Scopus and Google Scholar

- Research which is freely available for redistribution 